\title{
IAC-02-Q.4.2.03
}

\section{Venus Atmospheric Exploration by Solar Aircraft}

G. A. Landis

NASA John Glenn Research Center, Mailstop 302-1, Cleveland, $\mathrm{OH} 44135$

geoffrey.landis@grc.nasa.gov

C. LaMarre

University of Illinois, Department of Aeronautical Engineering, Champaign IL

A. Colozza

Analex Engineering, NASA Glenn Research Center, Cleveland $\mathrm{OH} 44135$

\section{3rd International Astronautical Congress The World Space Congress - 2002 10-19 Oct 2002/Houston, Texas}

For permission to copy or republish, contact the International Astronautical Federation 3-5 Rue Mario-Nikis, 75015 Paris, France 


\title{
VENUS ATMOSPHERIC EXPLORATION BY SOLAR AIRCRAFT
}

\author{
Geoffrey A. Landis \\ NASA John Glenn Research Center, Mailstop 302-1, Cleveland, OH 44135 \\ geoffrey.landis(a) grc.nasa.gov \\ Christopher LaMarre \\ University of Illinois, Department of Aeronautical Engineering, Champaign IL \\ Anthony Colozza, \\ Analex Engineering, NASA Glenn Research Center, mailstop 301-3, Cleveland OH 44135
}

\begin{abstract}
The Venus atmosphere is a favorable environment for flying powered aircraft. The atmospheric pressure makes flight much easier than on planets such as Mars. Above the clouds, solar energy is available in abundance on Venus, and the slow rotation of Venus allows a solar airplane to be designed for flight within continuous sunlight. The atmosphere between $50 \mathrm{~km}$ and $75 \mathrm{~km}$ on Venus is one of the most dynamic and interesting regions of the planet. The challenge for a Venus aircraft will be the fierce winds and caustic atmosphere. In order to remain on the sunlit side of Venus, an exploration aircraft will have to be capable of sustained flight at or above the wind speed. An aircraft would be a powerful tool for exploration. By learning how Venus can be so similar to Earth, and yet so different, we will learn to better understand the climate and geological history of the Earth.
\end{abstract}

\section{INTRODUCTION}

Recently it has been proposed that an aircraft could be flown in the atmosphere of the planet Mars. This is a difficult problem, since the low density of the Martian atmosphere means that in order to fly, an aircraft must have extremely low weight, a high wing area, or a very high flight speed, compared to a terrestrial aircraft.

Although there have been many recent studies on an airplane mission on Mars, Mars is not the only candidate planet where flight is possible. Earth's other neighboring planet, Venus, offers an extremely favorable atmosphere for powered flight $[1,2]$.

There have been many exploration vehicles sent to Venus in the past, from the Soviet probes "Venera" [3, 4] and "Vega" [5] to the United States "Pioneer Venus" [3,4], shown in figure 1, and Magellan Missions [5]. All of these missions gathered information on the atmosphere of Venus, but they did so while at the mercy of Venus' gravity or wind. The probes took atmospheric measurements while they descended towards the planet's surface, and the Vega balloon took measurements while being dragged along by the wind at relatively the same altitude during the entire mission.

The Venus atmosphere is an excellent environment for flying powered aircraft. The moderate temperature and atmospheric pressure at the cloudtop levels makes flight much easier than on planets such as Mars.

An airplane would be the ideal vehicle for gathering atmospheric data over a wide range of locations and altitudes, while having the freedom to maneuver to regions of scientific interest [1]. Additionally, airplanes would offer an order of magnitude better radar imaging resolution than orbiters, and cover a larger area than probes.

\section{VENUS ENVIRONMENT}

Above the clouds, solar energy is available in abundance on Venus. Venus has an exoatmospheric solar flux of $2600 \mathrm{~W} / \mathrm{m}^{2}$, compared to Earth's $1370 \mathrm{~W} / \mathrm{m}^{2}$. The solar 
intensity is 20 to $50 \%$ of the exoatmospheric intensity (depending on wavelength) at the bottom of the cloud layer at $50 \mathrm{~km}$, and increases to nearly $95 \%$ of the exoatmospheric intensity at $65 \mathrm{~km}$, the top of the main cloud layer [6]. In addition, the slow rotation of Venus allows an airplane to be designed for flight within continuous sunlight, eliminating the need for energy storage for nighttime flight. At these altitudes, the temperature of the atmospheric is moderate, in the range of 0 to $100 \mathrm{C}$ depending on altitude [7].

The atmosphere between $50 \mathrm{~km}$ and $75 \mathrm{~km}$ on Venus is one of the most dynamic and interesting regions of the planet. The challenge for a Venus aircraft will be the fierce winds and caustic atmosphere. As seen in figure 2, at the altitudes of interest to flight,the wind reaches a speed of about $95 \mathrm{~m} / \mathrm{s}$ at the cloud top level [7], and in order to remain on the sunlit side of Venus, an exploration aircraft will have to be capable of sustained flight at or above the wind speed.

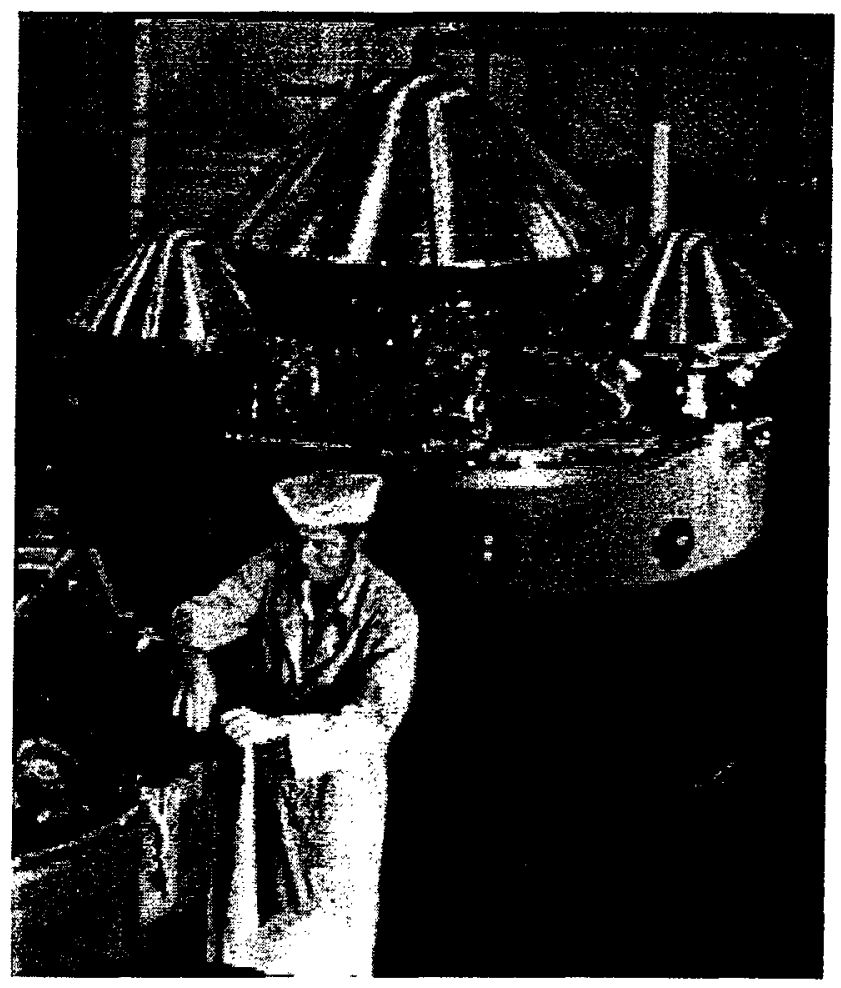

Figure 1: the Pioneer Venus spacecraft, carrying three small aeroshells and one large aeroshell for atmospheric entry.

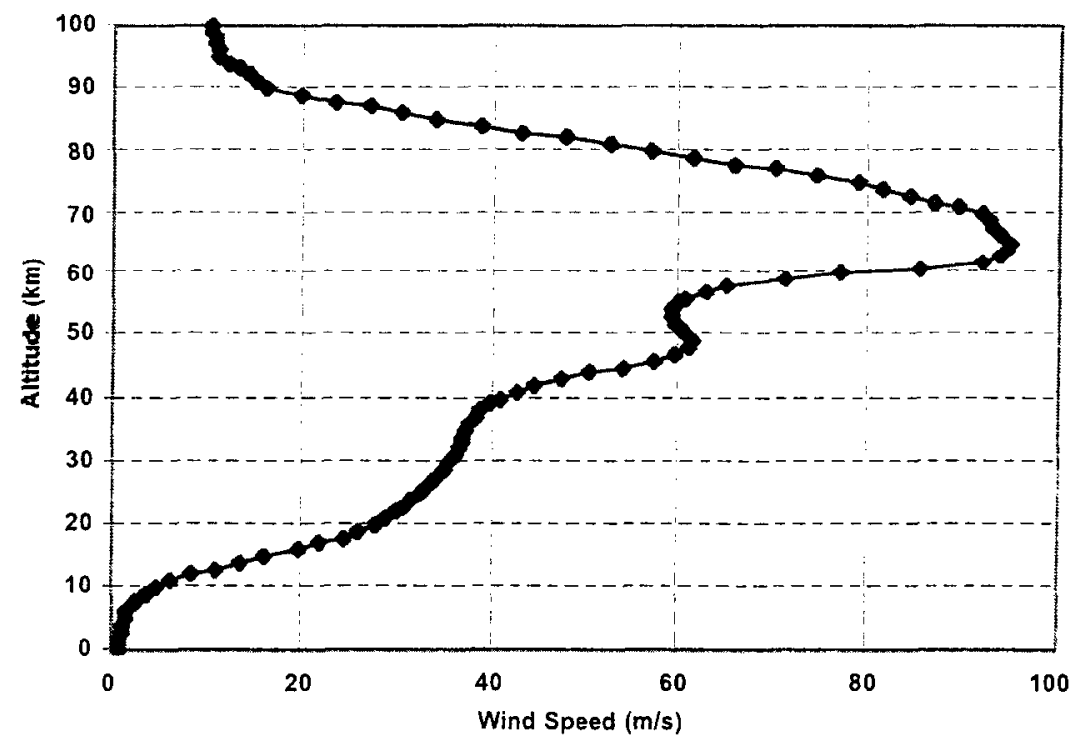

Figure 2: Wind speed as a function of Altitude on Venus 


\section{VENUS AIRCRAFT}

An aircraft sized to fit in a 1.3-meter diameter aeroshell for a "Discovery" class mission is proposed [2]. The aeroshell diameter is picked to be identical in size to that of the small atmospheric probes from the Pioneer Venus mission, shown in Figure 1 [from ref. 4]. For reliability, it is desirable that the number of moving parts be minimized. A candidate Venus airplane design is shown in unfolded flightconfiguration in figure 3 . The design requires only two folds to fit the wing into an aeroshell, and no folds to deploy the tail. Details of the design trade-offs are given in earlier work by Landis, Lamarre and Colozza [2].

Because of the design constraint that the two-fold wing is to fit into a small aeroshell, the wing area is maximum at extremely low aspect ratio, and higher aspect ratios can be achieved only by reducing the wing area. To fit a circular aeroshell, the resulting design trade-off increases wing area by accepting the design compromise of an extremely short tail moment and small tail area (stabilizer area $9 \%$ of wing area). In terms of flight behavior, the aircraft is essentially a flying wing design with the addition of a small control surface.

A more conventional aircraft design can be made by folding or telescoping the tail boom as well as the wing. Figure 4 shows an alternate concept, an inflatable-wing aircraft. Inflatablewing aircraft have been tested by NASA Dryden on the I-2000 aircraft [8.9], and offer the possiblity of a much larger wing being able to be stored into a small volume.

Typical flight altitudes for analysis were 65 to $75 \mathrm{~km}$ above the surface, at and slightly above the cloud layer. At this altitude, the atmospheric pressure is similar to pressure at terrestrial flight altitudes. For exploration of lower altitudes, it is feasible to glide down to low altitudes for periods of several hours, accepting the fact that the airplane ground track will blow downwind, and then climb back to higher altitudes and fly upwind to the original point, allowing both high and low altitudes to be probed.

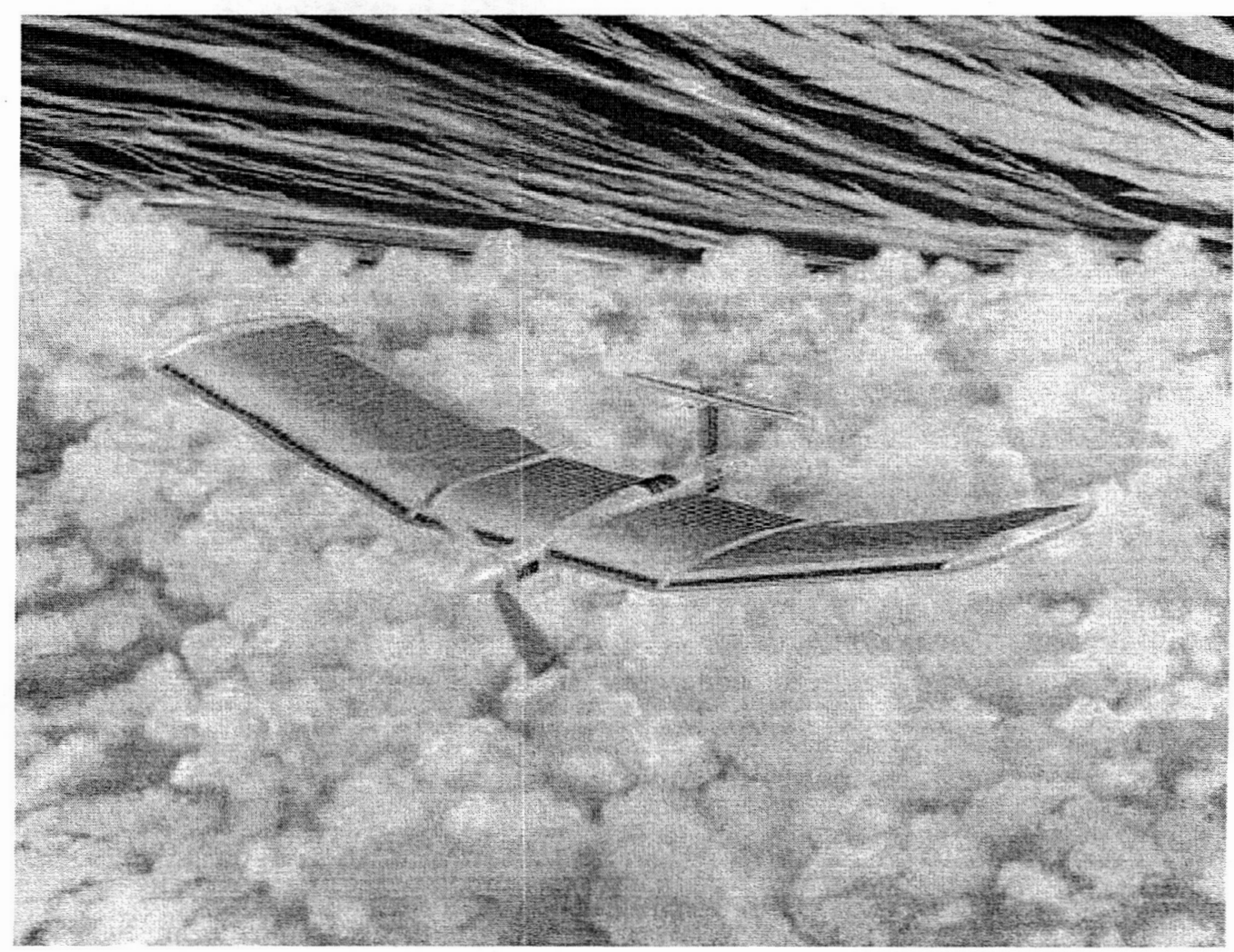

Figure 3: Artist's conception of a small solar airplane flying above the clouds in the Venusian atmosphere 


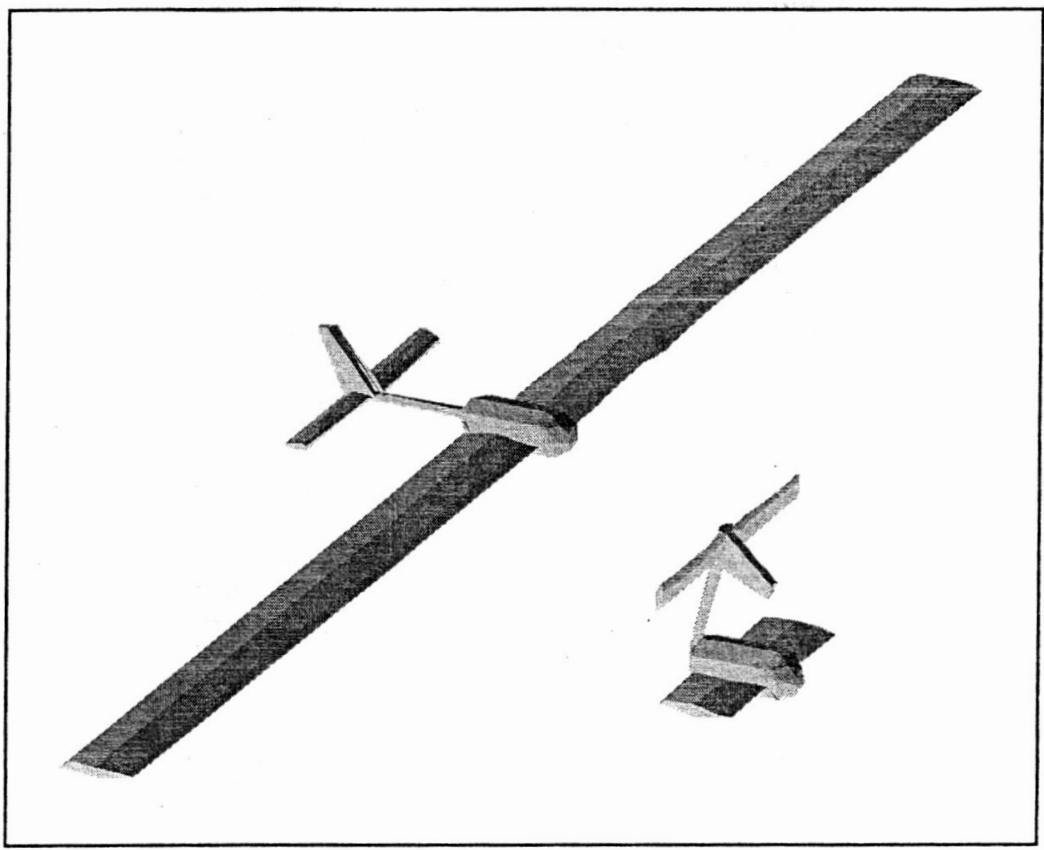

Figure 4: Deployment concept for a $n$ inflatable-wing aircraft.

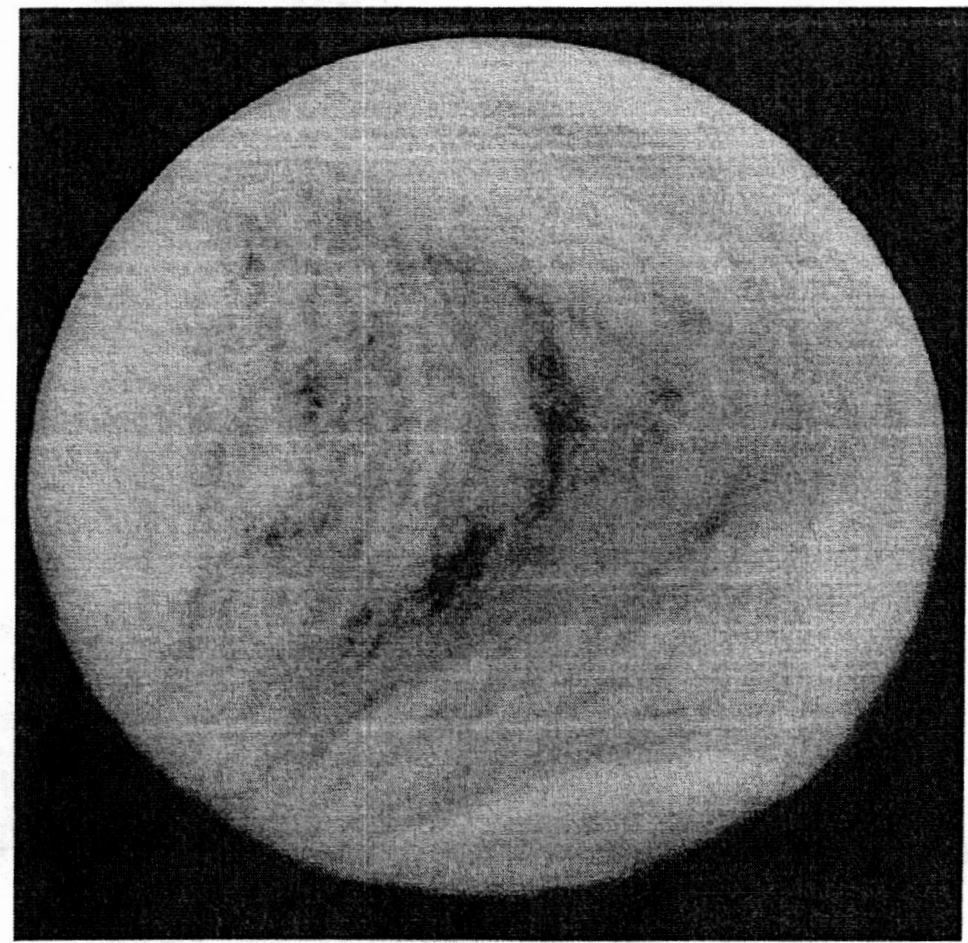

Figure 5: Venus, viewed in ultraviolet light from the Pioneer Venus spacecraft. 
Even at the highest wind areas of the planet, if the aircraft enters the nightside of the planet near the equator it will spend nearly two (terrestrial) days in darkness before re-emerging into the sunlit area. Analysis of flight using battery storage shows that it is not feasiblc to keep the aircraft aloft on battery power alone during the passage across the night side of the planet. Near the polar regions passage of the night region of the planet can be decreased in duration but in these regions the sunlight is incident on the aircraft at a high angle, and the solar arrays are significantly reduced in power output.

Likewise, the unpowered glide range of the aircraft is not high enough for it to glide around the night side of the planet and re-emerge into sunlight. Therefore, if the mission duration is to be unlimited, the mission is restricted to the daylight side of the planet, and to altitudes high enough that the aircraft can equal or exceed the wind speed.

\section{SCIENTIFIC MISSION OF VENUS AIRPLANE}

The planet Venus has a number of fundamental scientific mysteries, both in its atmosphere, and in its surface.

Crisp [10] calls the environment of Venus "among the most enigmatic in the solar system." He notes that, although the volatile inventory of Venus are not yet well characterized, existing measurements suggest that the relative abundances of the noble gases in its atmosphere are much more solar-like than those on the other two terrestrial planets. Similarly, although today Venus has an inventory of water that is a hundred thousand times less water than Earth, the deuterium to hydrogen ratio in the atmosphere is on the order of 150 larger for Venus than for Earth. Since hydrogen is more easily lost from the atmosphere than deuterium, this suggests that the original amount of water present on Venus may have been comparable or larger than that on Earth. The detailed process and timing of this large loss of water are not known, leaving a large blank area in efforts to understand the history and change of the surface and atmosphere.
An aircraft mission to the atmosphere of Venus would have the ability to sample the atmosphere at a variety of different locations, including both different latitude bands and also different altitudes, thus sampling the volatile inventory as a function of location and altitude.

The cycle of sulfur in the Venus atmosphere is not yet understood. The sulfur interacts with the surface minerals, which serve as a repository of sulfur. Are there presently-active sources of atmospheric sulfur, in the form of volcanoes or gas vents? By "sniffing" the sulfur content of the atmosphere as a function of position on the surface, an aircraft could be a vehicle to determine whether the sulfur abundance in the atmosphere is correlated with specific surface features (i.e., volcanoes or fumaroles).

Atmospheric dynamics of Venus are also not yet understood. Although the surface of Venus rotates very slowly, with a 242 day period, the atmosphere at the cloud level and higher moves around the planet a period of 4 days, 60 times faster than the surface. The question of exactly what mechanism supports this atmospheric super-rotation is a question that has eluded detailed explanation since its discovery by spacecraft in the early 1970s. In order to address this problem, an aircraft should measure the infrared absorption in the atmosphere, as well as measuring the horizontal and vertical components of atmospheric motion as a function of altitude, latitude, and subsolar longitude. Detailed modeling of the atmospheric will also require knowledge of the atmospheric correlation, which can be done by use of two aircraft flying with known separation in both vertical and horizontal directions.

Even the nature of the absorbing particles in the clouds remains enigmatic. This could be measured by chemical and physical sensors in the atmosphere.

Finally, Venus is the best laboratory in the Solar system for study of the Greenhouse effect. If we are to understand climate change on Earth, we can learn by comparing and contrasting the history and climate of the Earth with that of its nearby neighbors, Venus and Mars. A measurement of infrared absorption, reflection, and emission in the atmosphere of Venus will do much toward elucidating the greenhouse effect mechanisms on Venus, and allowing us to compare and contrast the 
greenhouse effect on Venus with that of Mars and Earth.

The most exciting discovery remaining to be made in the solar system would be the discovery of life. While the surface of Venus is extremely hostile to any hypothesized form of life, the atmosphere of Venus is in many ways one of the most hospitable environments outside of the Earth for biology. At the cloud-top level, the temperature and pressure of Venus' atmosphere is nearly terrestrial, and water is present in the form of sulfuric acid droplets. According to Schulze-Makuch [11], chemical disequilibrium in the atmosphere of Venus is a possible signature of microbiological activity. Data on Venus from the Venera missions and the Pioneer Venus and Magellan probes shows that carbon monoxide is scarce in the planet's atmosphere, although solar radiation and lightning should produce it abundantly. Hydrogen sulfide and sulfur dioxide, two gases which react with each other thus should not be found together, are also both present, possibly indicating something is producing them. Finally, although carbonyl sulfide is difficult to produce inorganically, it is present in the Venusian atmosphere. On Earth, this gas would be considered an unambiguous indicator of biological activity. According to SchulzeMakuch, these chemical indicators are a possible indicator of biological activity in the Venusian cloud layers that could be combining sulfur dioxide with carbon monoxide and hydrogen to produce hydrogen sulfide and carbonyl sulfide.

Aircraft-borne instrumentation could examine in detail the chemical composition of the atmosphere as a function of altitude, and also could be used to directly search for the putative microorganisms.

The surface of Venus is also of great scientific interest. Crisp [10] notes that the surface of Venus has a number of scientific enigmas that have yet to be elucidated. Estimates from the density of impact craters indicates an average surface age of 500 to 700 million years [5]. What has caused this resurfacing of the planet, quite recent on the time scale of geological time? Venus lacks large tracts of young and old crust, a signature of Earth's plate tectonics cooling mode. Venus also lacks evident plate boundary features and other evidence of plate tectonics.
In addition, the mountain tops of Venus have some indication of interesting mineralogy that is different from the lower altitudes. The nature of this mineralogy is unknown.

The primary tool for aircraft-based investigation of the surface of Venus would likely be radar. By observing the surface from a distance of tens of kilometers, rather than the hundreds of kilometer altitude of an orbiting probe, resolution will be improved by a factor of ten, and the required power will be reduced by a factor of a hundred. An aircraft will also be able to circle over a particular spot of interest, allowing detailed study of one spot, rather than uniformly covering all regions of the planet.

An aircraft radar investigation could also be done in the bistatic mode with a variable angle between the transmitter and receiver. This could be done either by having one aircraft transmit while one or more different aircraft receive the signal, or also be done by having the transmitted radio pulse originate from an orbital probe (or, inversely, a signal originating from an aircraft could be received by an orbiting receiver.)

Overall, many scientific investigations are possible from an aircraft based platform. that could allow us to expand our knowledge of Venus. coordinated observations of Venus, Earth, and Mars to understand the similarity and differences in the evolution and history is essential to developing a significant understanding of the origin and evolution of Earth-like terrestrial planets.

\section{CONCLUSIONS}

An aircraft, with the ability to control its position in the atmosphere of Venus instead of drifting helplessly with the wind, would be a powerful tool for exploration. By learning how Venus can be so similar to Earth, and yet so different, we will learn to better understand the climate and geological history of the Earth. The success of a prototype solar airplane could lead to the development of a fleet of solarpowered airplanes flying across the Venus cloud tops, taking simultaneous measurements to develop a "snapshot" of the climate across the face of the planet. Fleets of solar-powered aircraft could provide an architecture for efficient and low-cost comprehensive coverage for a variety of scientific missions, both 
atmospheric and geological science via surface imaging and radar. Exploratory planetary mapping and atmospheric sampling can lead to a greater understanding of the greenhouse effect not only on Venus but on Earth as well.

\section{REFERENCES}

1. G. Landis, "Exploring Venus by Solar Airplane," presented at the STAIF Conference on Space Exploration Technology, Albuquerque NM, Feb. 11-15, 2001. AIP Conference Proceedings Volume $552,16-18$.

2. G. Landis, C. LaMarre and A. Colozza, "Atmospheric Flight on Venus," paper AIAA-2002-0819, AIAA $40^{\text {th }}$ Aerospace Sciences Meeting, Reno NV, January 14-17 2002. NASA Technical Memorandum 2002211467 (2002).

3. D. M. Hunten, L. Colin, T. M. Donahue, and V. I. Moroz, eds., Venus, University of Arizona Press 1983.

4. R. Fimmel, L. Colin, and E Burgess, Pioneer Venus, NASA SP-461, 1983.

5. S. Vougher, D. Hunten, and R. Phillips, eds., Venus II, University of Arizona Press, 1997.
6. A. Ekonomov, Yu. Golovin, V. Moroz and B. Moshkin, "19: Solar Scattered Radiation Measurements by Venus Probes," in D. M. Hunten, L. Colin, T. M. Donahue, and V. I. Moroz, eds., Venus, University of Arizona Prcss 1983.

7. A. Seiff, "Thermal Structure of the Atmosphere of Venus," in D. M. Hunten, L. Colin, T. M. Donahue, and V. I. Moroz, eds., Venus, University of Arizona Press 1983.

8. J. Murray, J. Pahle, S. Thornton, S. Vogus, T. Frackowiak, J.. Mello, and B. Norton, "Ground and Flight Evaluation of a SmallScale Inflatable-Winged Aircraft," 40th AIAA Aerospace Sciences Meeting and Exhibit, 14-17 Jan. 2002, Reno, NV

9. NASAexplores: February 14, 2002. http://nasaexplores.com/lessons/02-013/

10. D. Crisp, "Divergent Evolution Among Earth-like Planets: The Case for Venus Exploration," Planetary Decadal Study Community White Paper, Solar System Exploration Survey, 11/21/01

11. D. Schulze-Makuch, 2nd European Workshop on Astrobiology, Graz, Austria, 16-19 Sept 2002. Reported in New Scientist, 25th September 2002. 